\title{
Sudden Unexpected Death in Alcohol Misuse: A Case Report
}

\author{
Ranil HK Sanjeewa ${ }^{1 *}$, D Gey van Pittius ${ }^{1}$, Muditha Vidanapathirana ${ }^{2}$
}

${ }^{1}$ Department of Pathology, Royal Stoke University Hospital, Stoke on Trent United Kingdom.

${ }^{2}$ Department of Forensic Medicine, Faculty of Medical Sciences, University of Sri

Jayewardenepura, Sri Lanka.

Alcohol is associated with a significant number of sudden deaths per year in the UK and deaths from alcohol-related causes are increasing at an alarming rate. These deaths are more common among white men who are over 50 years with fatty changes rather than the cirrhotic liver. The only findings at postmortem are fatty liver and a negative or low blood alcohol level. The mechanism of death is uncertain in most of the instances but there are some theories related to the suggested mechanism. Sometimes, pre-existing minor causes are incorrectly diagnosed as the cause of death. Therefore, forensic pathologist experiences major difficulties in ascertaining the cause of death. Awareness of the association between alcohol-related fatty change and sudden death is essential to give a correct cause of death at the autopsy examination. A 63-year-old man was found dead in his bathroom by his partner. There was no past medical history of diabetes mellitus, hypertension and alcohol withdrawal. He consumed 5-6 bottles of wine per day over the past 9 months. Postmortem examination revealed slight jaundice, pallor, mild atheromatous changes in coronary arteries, mild left ventricular hypertrophy and marked fatty changes in the liver. Microscopic examination of the liver revealed severe steatosis, predominantly macrovesicular. In addition to that, there was no evidence of chronic disease or pathology. Toxicology analysis revealed $113 \mathrm{mg} / \mathrm{dl}$ of blood alcohol reset of investigations were unremarkable. Limited biochemical analyses were done. They did not show any evidence of significant electrolytes imbalances or hypo or hyperglycemia. Excluding all the possibilities and considering macroscopic and microscopic features, and toxicological investigations, the cause of death was ascertained as sudden unexpected death in alcohol misuse (SUDAM).

Keywords: SUDAM, Alcohol misuse, Diagnosis by exclusion

Copyright: @ 2018 with the Medico-legal Journal of Sri Lanka.

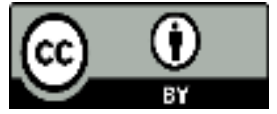

This is an open-access article distributed under the terms of the Creative Commons Attribution License, CC BY 4.0 which permits unrestricted use, distribution and reproduction in any medium, provided the original author and source are credited.

Funding: None

Competing interests: None

Received: 01 April 2018 Accepted revised version: 01 May 2018 Published: 30 June 2018

*Corresponding author: Sanjeewa HKR, Email: sanjweewa2307@yahoo.com $\square \quad$, Tel: +94777705997 https://orcid.org/0000-0003-4958-6009

Cite this article as: Sanjeewa HKR, Pittius DGV, Vidanapathirana M. Sudden unexpected death in alcohol misuse: a case report. Medico-Legal Journal of Sri Lanka, 2018;6(1): 43-46.

DOI: http://dx.doi.org/10.4038/mljsl.v6i1.7373 


\section{Introduction}

Alcohol is associated with approximately 40,000 deaths per year in the UK and deaths from alcoholrelated causes are increasing at an alarming rate, having increased at least eightfold since the 1970s. ${ }^{[1]}$

An association between fatty changes within the liver due to alcohol and sudden cardiac arrhythmic deaths was described in $1926{ }^{[2,3]}$

Sudden death can occur in chronic misuse of alcohol. ${ }^{[2,3]}$ The only findings at the postmortem are fatty liver and a negative or low blood alcohol level $[2,3]$. These deaths are more common among white men who are over 50 years with fatty changes rather than the cirrhotic liver.

The mechanism of death is uncertain in most of the alcohol-related sudden deaths but there are some formulated theories related to the mechanism. The metabolic disturbances are caused by alcohol intake and starvation are recognized causes and they lead to cardiac arrhythmia. ${ }^{[4]}$

Alcohol can be a cause for sudden cardiac death, but it cannot be identified by many pathologists ${ }^{[5]}$ in most of the instances or they misdiagnose as preexisting other minor causes such as minor coronary artery atherosclerosis or left ventricular hypertrophy. ${ }^{[6]}$

Therefore, forensic pathologists have experienced major difficulties giving conclusion during their postmortem examination.

In our case, we discuss how to ascertain the cause of death with available history, examination and investigation findings in an alcoholic who died suddenly.

\section{Case report}

A 63-year-old man was found dead on the floor of the bathroom by his partner. When the diseased was last seen by his partner earlier in same evening before going to work, he had not complained of feeling unwell. Later, when his partner phoned him, he had informed that his throat was sore due to vomiting. His partner returned home from work about six hours later in the night and found him lying on the bathroom floor. He was confirmed dead despite the paramedics' Cardiac Pulmonary Resuscitation (CPR).

There was no significant past medical history of diabetes mellitus, hypertension and alcohol withdrawal. He was a smoker and had stopped about 30years ago. He had not taken any illegal drugs but he had consumed numerous bottles of wine over the past 9 months (He consumed 5-6 bottles of wine per day).

The postmortem examination did not reveal any injury in clothes or the body. Slight jaundice and pallor were evident. Coronary arteries had mild atheromatous changes. Left ventricular wall thickness was $1.5 \mathrm{~cm}$. Marked fatty changes were evident in the liver.

The histopathological examination of the lungs showed congestion and mild patchy inflammation but no evidence of bronchitis or bronchopneumonia. A single small artery contained a thrombus. The heart showed patchy myocyte hypertrophy with no evidence of inflammation or infarction including contraction band necrosis (Fig. 1). The kidneys showed autolysis and mild patchy inflammation with no interstitial inflammation, necrosis or glomerulonephritis.

The liver showed severe steatosis, macrovesicular and also microvesicular with no evidence of cirrhosis, malignancy, inflammation or necrosis. The predominant macrovesicular fatty change was evident as eccentric nuclei, swelling of hepatocytes and compression of sinusoids with pavement like pattern. (Fig. 2). The scanty microvesicular pattern was evident as foamy degeneration. The toxicology analysis revealed that the blood alcohol level was $113 \mathrm{mg} / \mathrm{dl}$ and it does not show any significant abnormalities related to any other therapeutic or illegal drugs or poisons.

The limited biochemical analysis did not show evidence of hypo or hyperglycemia or electrolytes imbalances.

There were no macroscopic or microscopic evidence suggestive of any other pathology or diseases.

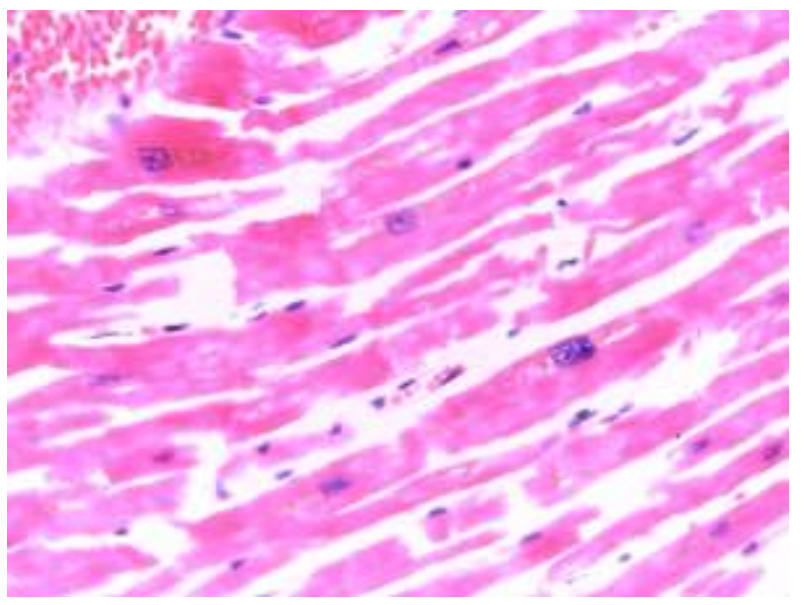

Figure 1: Hypertrophy of the myocytes (H \& E 40) 


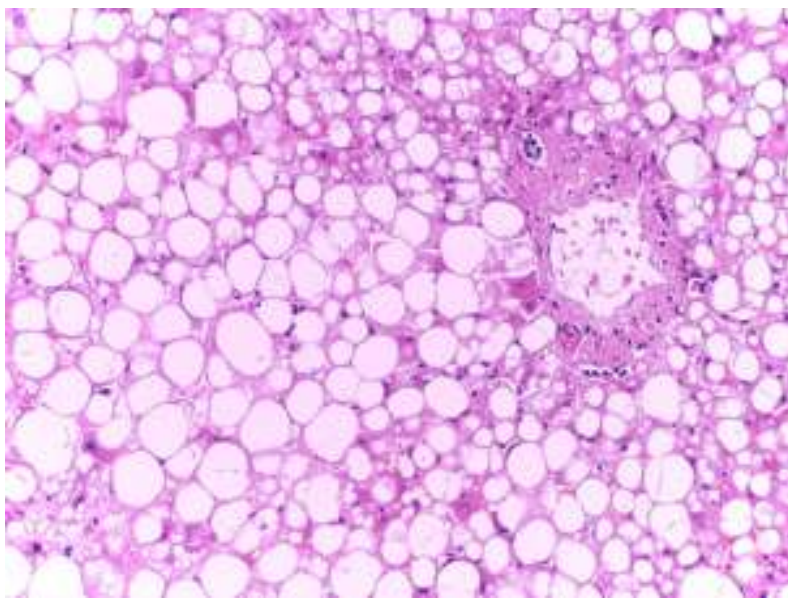

Figure 2: Sever macrovesicular steatosis (H \& E 80)

\section{Discussion}

Heart showed mild left ventricular hypertrophy but no evidence of past ischaemic changes such as myocardial fibrosis or acute ischaemic changes or contraction band necrosis or infarctions. Further, there was no significant coronary artery disease. Myocardial hypertrophy can lead to cardiac arrhythmias and sudden death by causing ischaemia or abnormalities in the conduction of electrical impulses. $^{10}$

There were no macroscopic or microscopic evidence of acute significant pathology in the lungs, kidneys or brain.

The rest of the organs were unremarkable and there were no identifiable chronic diseases or pathology. The liver showed severe yellow discolouration and histology confirmed hepatic steatosis in keeping with the history of excess alcohol consumption. It is the most reliable evidence for high consumption of alcohol more than $80 \mathrm{~g}$ or 10 units of alcohol per day. ${ }^{[7]}$

Toxicological analyses of body fluids were unremarkable and there was no evidence of illegal or therapeutic drugs or poisons. We had done limited biochemical analysis such as electrolytes and blood sugar level. They were unremarkable. Commenting about hypoglycemia is very difficult due to the limitation of available samples such as antemortem sugar level.

Sudden death is more common in chronic alcohol misuse. The relationship between sudden death and fatty liver has been confirmed in a number of studies which have reported a link between fatty liver and death in chronic alcoholics. ${ }^{[2.3]}$ The mechanism(s) of death in these are uncertain. Possibilities include fat embolism, alcohol withdrawal, hypoglycemia, alcoholic ketoacidosis and other electrolyte abnormalities. Cardiac arrhythmia is most favoured as the likely mechanism. Many alcoholics have a prolong QT-interval which is associated with an increased risk of sudden death. ${ }^{[8]}$ Chronic alcoholism and fatty liver is associated with profound electrolyte disturbances including hypomagnesaemia which can lead to fatal arrhythmia. ${ }^{[5]}$ The term SUDAM (sudden unexpected death in alcohol misuse) has been coined recognizing the increased risk of sudden death in alcoholics. ${ }^{[2,3,4,5,8]}$ SUDAM is applied to sudden unexpected non-traumatic deaths where there is a history of chronic excess alcohol consumption and/or evidence of hepatic steatosis but no other anatomical or toxicological cause of death and no significant cardiac hypertrophy. ${ }^{[2,3,4,5]}$. Then, we have excluded all the possibilities and come to the conclusion. Therefore it will be diagnosed by exclusion.

\section{Conclusion}

Considering the autopsy examination findings including histological examination and toxicology; no obvious pathological, biochemical and toxicological finding was identified. The main significant histology is fatty steatosis. There was no history of foul play, suicidal or homicidal activities. Excluding all the possibilities and considering macroscopic and microscopic features and toxicological investigations, the most likely cause of death was concluded as sudden unexpected death in alcohol misuse (SUDAM).

\section{References}

1. Leon DA, McCambridge J. Liver cirrhosis mortality rates in Britain, 1950 to 2002. Lancet. 2006;367 (9511):52-56. PMID:16503452

2. LeCount ER, Singer HA. Fat replacement of the glycogen in the liver as a cause of death. 1926. Arch Pathol Lab Med. 2001;125(1):1520.

DOI:10.1043/0003-985(2001)125<0015: HP> 2.0.CO;2

3. Chejfec G. Fat replacement of the glycogen in the liver as a cause of death: seventy-five years later. Arch Pathol Lab Med. 2001;125(1):21-4. DOI:10.1043/00039985(2001)125<0021:FRO TGI $>2.0 . \mathrm{CO} ; 2$

4. Yuzuriha T, Okudaira M, Tominaga I, Hori S, Suzuki H, Matsuo Y et al. Alcohol-related sudden death with hepatic fatty metamorphosis: a comprehensive clinicopathological inquiry into its pathogenesis. Alcohol Alcohol. 1997;32(6):745-52.

PMID:9463729 
5. Randall B. Fatty liver and sudden death. A review. Hum Pathol. 1980;11(2): 147-53. PMID:6105125

6. Petersson B. Analysis of the role of alcohol in mortality, particularly sudden unwitnessed death in middle-aged men in Malmo Sweden. Alcohol Alcohol. 1988;23(4):259-63. PMID:3166624

7. Karhunen PJ, Penttila A. Validity of postmortem alcohol reports. Alcohol Alcohol. 1990;25(1):25-32.

PMID:2334492
8. Day CP, James OF, Butler TJ, Campbell RW. QT prolongation and sudden cardiac death in patients with alcoholic liver disease. Lancet. 1993;341:1423-8.

PMID:8099138

9. Templeton AH, Carter KLT, Sheron N, Gallagher PJ, Verrill C. Sudden unexpected death in alcohol misuse - an unrecognized public health issue? Int J Environ Res Public Health. 2009;6:3070-3081.

Doi: http//dx.doi.org/10.3390/ijerph6123070.

10. Mary N, Practical Cardiovascular Pathology. Boca Raton: Taylor \& Francis; 2000: 24-66. 